\title{
Fortalecimiento del Vínculo Padres-Hijo a Través del Proceso de Cuidado de Kristen Swanson
}

\section{Enhancement of the Bond Between Parents-Child Through the Care Process by Kristen Swanson}

Recibido 1 Ago. 2013/Enviado para Modificación 30 Sep. 2013/Aceptado 15 Nov. 2013

Merilyn Guerra Ramírez ${ }^{1}$

Universidad Simón Bolívar

\section{RESUMEN}

El presente artículo invita a la reflexión sobre la importancia de fomentar el vínculo entre los padres y sus hijos hospitalizados en las unidades de cuidado intensivo neonatal. Teniendo en cuenta que la vinculación afectiva se define como una relación única, específica y perdurable entre dos personas, la cual tiene efectos profundos en el desarrollo físico, psicológico e intelectual, y que dicha relación suele verse afectada por las exigencias y barreras que impone el soporte tecnológico complejo de las unidades de cuidados intensivos, se busca integrar al ejercicio de la profesión, en la práctica diaria, los postulados de la teoría expuesta por Kristen Swanson y su proceso de cuidado, con el fin de establecer un cuidado de Enfermería que favorezca la participación de los padres como miembros del equipo multidisciplinario. De esta forma, se reconocen y potencian sus capacidades en el cuidado del niño, y, por otro lado, se aclaran sus dudas y se les brinda atención y educación personalizada.

Palabras Clave: Vínculo, cuidado de Enfermería, teoría de Enfermería, Unidad de Cuidado Intensivo Neonatal. (Fuente: DeCS).

\begin{abstract}
This paper focuses on the importance of promoting the relationship between parents and their children admitted in the Neonatal Intensive Care unit. Keeping in mind, that the emotional bonding is considered as a unique, specific and lasting relationship between two people; this one, has deep effects on the physical, psychological and intellectual development, and this relationship could be affected by the requirements and barriers imposed by the complex technological support of the intensive care unit, then it aims to involve the practice of the profession in the daily practice. The postulates of the theory about care process by Kristen Swanson, propose to establish a nursing care which promotes to involve the parents as members of the multidisciplinary team. In this way, their skills are recognized and enhance in the child's care, and in the other hand, the doubts are clarified giving them attention and personalized education
\end{abstract}

Keywords: Bonding, Nursing Care, Nursing theory, Neonatal Intensive Care Unit (Source: MeSH, NLM).

1 Enfermera. Magister en Enfermería. Docente Universidad Simón Bolívar. Enfermera Unidad de Cuidado Intensivo Neonatal Clínica El Prado, Barranquilla-Colombia. Correo electrónico: mguerra@unisimonbolivar.edu.co. 


\section{Introducción}

En años recientes, la atención humanizada al interior de las Unidades de Cuidados Intensivos Neonatales (UCIN) ha tomado fuerza, y la profesión de Enfermería no es ajena a este cambio. La atención en este campo se centra en la resolución de morbilidades orientadas por el ordenamiento médico, pero este suele apegarse a protocolos de atención institucionales $\mathrm{y}$ descuida los potenciales beneficios del cuidado "no farmacológico" de los padres. En este sentido, la cotidianidad de la práctica ha desdibujado los postulados de la teoría de Kristen Swanson (Teoría de los Cuidados), la cual propone que, a través de la práctica simple de cinco procesos (conocer, hacer por, estar con, posibilitar y mantener las creencias), se genere el bienestar de la atención más allá de las intervenciones sanitarias. Lo anterior hace que dicha teoría se convierta en una posible guía de cambio en el paradigma de atención humanizada orientado al fortalecimiento del vínculo padres-hijo hospitalizado en UCIN, pues, dichos conceptos permiten reflexionar sobre la cosmovisión del cuidado del enfermero (a), en quien convergen las dimensiones históricas, antropológicas y filosóficas de la ciencia de Enfermería. Teniendo en cuenta que para Swanson la Enfermería significa cuidado informado para el bienestar del otro (en este caso padres, hijo y familia), este cuidado se informa mediante el conocimiento empírico a partir de la Enfermería y de ciencias afines (humanidades), experiencias clínicas y valores personales y sociales de las personas (1). Así entonces, la Enfermería es la disciplina conocedora de los cuidados para el bienestar de los padres y sus hijos.

Pero es importante reflexionar sobre el proceso de cuidado de Enfermería, el cual trasciende la mejora de una persona per se, y la asiste para alcanzar, mantener o recuperar el nivel óptimo de bienestar requerido para responder a las demandas que se le hacen, sortear obstáculos, y utilizar los recursos personales y del entorno (2). En este orden de ideas, es necesario conocer e integrar los postulados de la teoría a nuestra práctica diaria, ya que las teorías son las que permiten la generación del conocimiento $\mathrm{y}$, de esta manera, avanzar en la conformación de un cuerpo propio de estructuras disciplinares, y de obtener un soporte filosófico que sustente la práctica.

De acuerdo con los planteamientos anteriores, el fortalecimiento del vínculo padres-hijo, a través del proceso de cuidado, se constituye en una forma educativa de relacionarse con un ser apreciado hacia el que se siente un compromiso y una responsabilidad personal. Independientemente de la experiencia y de los años de ejercicio laboral del profesional de Enfermería, el fortalecimiento del vínculo padres-hijo aparece como un conjunto de procesos secuenciales, creados por la propia actitud filosófica de Enfermería, la cual establece cinco categorías o procesos que se presentan de manera separada, pero que constituyen una secuencia de intervenciones propias de la profesión. El objetivo central del presente artículo de reflexión consiste justamente en llamar la atención sobre el vínculo padres-hijo al interior de las UCIN, a través del proceso de cuidado.

\section{Desarrollo}

La vinculación afectiva se define como una relación única, específica y perdurable entre dos personas, la cual tiene efectos profundos en el desarrollo físico y psicológico, así como en el desarrollo intelectual, durante los primeros años de vida y se constituye, además, en el fundamento de la relación padres-hijo que se desarrolla a lo largo del tiempo (3).

Durante este proceso de vinculación, es importante que el niño responda con comportamientos que refuercen la socialización, tales como la sonrisa y la mirada a los ojos de sus padres. Por esto, cuando hay una separación prolongada del trinomio, debido a la hospitalización del niño en una UCIN, se provoca en los padres una ruptura de roles, angustia elevada y una pérdida de control sobre la situación. Esta situación y los sentimientos que la acompañan provocan, a su vez, una pérdida de autonomía, al depender en mayor o menor grado del equipo de salud que cubre las necesidades de su hijo. En estas circunstancias, los padres abandonan sus rutinas diarias, trabajo, amigos, familia, y su única razón de vida se centra en acompañar a su hijo de lejos, en una sala de espera en la que a veces se vuelven invisibles, afectando, en consecuencia, el vínculo y trayendo como consecuencia trastornos en el desarrollo mental, motor y afectivo del niño (4).

Por otra parte, las diversas condiciones que dificultan el proceso de adaptación a la vida extrauterina y algunos procesos mórbidos que pueden presentarse en el neonato $(5,6)$, hacen necesaria su vigilancia cuidadosa y permanente, lo que motiva la

Cienc. innov. salud. Diciembre 2013; 1 (2):120 - 125. Universidad Simón Bolívar (Col). ISSN: 2344-8636 http://portal.unisimonbolivar.edu.co:82/rdigital/innovacionsalud 
hospitalización en la mayoría de los casos (7). A esto se suma el hecho de que las madres de estos niños experimentan altos niveles de estrés y emociones negativas, tales como ansiedad, depresión, culpa y desesperanza, y dado que las circunstancias a las que están sometidas son intensas, esta ansiedad y preocupación terminan prolongándose en el tiempo (8).

Es de tener en cuenta, igualmente, que si se evalúan las condiciones de inmadurez y de bajo peso, que por lo general acompañan a la mayoría de los niños que requieren los servicios de la UCIN, lo más probable es que la hospitalización genere una separación forzosa y prolongada de sus padres, los cuales sufren con frecuencia sentimientos de culpa y depresión, dada las implicaciones sociales que supone el rol de padre; ya que, aun cuando deseen permanecer al lado de sus parejas y de sus hijos, deben regresar a sus compromisos laborales, interrumpiéndose así la formación del vínculo padres-hijo, y creándose distanciamientos que dificultan el comienzo de una relación de apego con el hijo (9).

Por todo lo planteado, en las últimas décadas, las tendencias en cuidado de Enfermería se han empeñado en transformar el modelo tradicional de asistencia centrado en el neonato enfermo, por un nuevo modelo, que permite la participación de la familia en la atención integral, a partir de nuevas filosofías, conceptos y teorías de cuidado (10).

Sin embargo, a pesar de los avances en la investigación y de la relevancia que han alcanzado los derechos del niño, la situación del neonato no ha cambiado en nuestro contexto (11). Efectivamente, en la mayoría de las instituciones de salud, la visita de los padres a los recién nacidos hospitalizados en UCIN aún se restringe y se controla mediante normas rígidas. Las barreras y los desafíos del medio físico (monitores, incubadoras, lámparas, respiradores), las reglas informales y formales (horarios de visita, forma de vestirse al ingresar a la unidad, lavado de manos, utilización de ropa especial) que se deben realizar para visitar al neonato son características ambientales que moldean la manera en que los padres se desempeñan, y limitan, de esta manera, su participación en el cuidado del neonato $(12,13)$. Aunque los padres no compartan tales políticas, entienden que estas normas institucionales van encaminadas a la protección de sus hijos y, por lo tanto, se ajustan a ellas $(14,15)$, ubicando en el personal de Enfermería el punto de referencia para el apoyo afectivo, y convirtiéndolos en un enlace clave entre ellos y su hijo por ser el personal que permanece mayor tiempo y establece mayor contacto con los pacientes. Pero no sobra decir que el equipo de Enfermería percibe todo esto como una responsabilidad adicional, poco deseada (16).

Urge, entonces, que el profesional de Enfermería se concientice respecto a la importancia que tiene el fomento del cuidado básico y el afecto de los padres a sus hijos: que abogue por la flexibilización de las normas institucionales existentes, y propicie así un ambiente de calidez y confianza, a fin de que los padres expresen sus sentimientos, manifiesten libremente sus preocupaciones, hagan preguntas, exploren opciones, y conozcan sus necesidades y sus preferencias al momento de contactar con sus hijos hospitalizados (17). Todo ello, fundamentando la práctica diaria en el conocimiento disciplinar, dando lugar a propuestas y políticas desde la Enfermería, que permitan la articulación de la teoría y la práctica en pro del fortalecimiento del vínculo padres-hijo al interior de nuestras unidades de cuidado crítico (18).

De igual forma, cabe resaltar que a pesar de las tendencias innovadoras en el cuidado humanizado de Enfermería $(19,20)$, presentes en nuestra práctica diaria, aún nos limitamos a manejar el estado patológico (21), según guías y protocolos planteados desde lo eminentemente biológico, minimizando riesgos para prevenir complicaciones. Además, es cuestionable la gestión de procesos administrativos, que, de manera absurda, desvirtúan la acción propia del cuidado, segmentando la integralidad biopsicosocial y espiritual del ser humano, al brindar un cuidado fraccionado de Enfermería.

Por tal razón, la Enfermería debe propender por el fortalecimiento del vínculo padres-hijo y por mejorar su práctica, basándose en el conocimiento de la disciplina, con el fin de establecer un cuidado que favorezca la participación de los padres como miembros del equipo multidisciplinario $(22,23)$, aclarando sus dudas, reconociendo y potenciando sus capacidades para el cuidado del niño, y brindándoles atención y educación personalizada $(24,25)$.

La necesidad de cambiar el paradigma de cuidado fraccionado por el de cuidado integral, obliga a entender el significado del cuidado como fenómeno de interés para la Enfermería. Precisamente, en su teoría del cuidado, Kristen Swanson define este concepto 
como "una forma de alimentar la relación de una persona valiosa hacia la cual se tiene un sentimiento personal de compromiso y responsabilidad" (26).

El cuidado, según Swanson, involucra cinco procesos terapéuticos: Conocer (tratando de comprender la experiencia del otro); estar con (hallarse emocionalmente presente); hacer por (como el otro lo haría para sí mismo, si fuera posible), posibilitar (facilitando la resolución, validando e informando), y mantener las creencias (en el potencial de otros para llegar al evento o transición y enfrentar un futuro significativo). Extrapolados a la práctica diaria de Enfermería, estos cinco procesos se evidencian en la actitud filosófica de la enfermera (o), la comprensión, los mensajes verbales y no verbales, las acciones terapéuticas y las consecuencias de los cuidados (27). En otro apartado, Swanson afirma que un componente fundamental y universal de la buena enfermería consiste en cuidar del bienestar físico, espiritual, sicológico y social de las personas, afirmación que evidencia en su teoría de los cuidados, al explicar lo que significa el modo de cuidar en la práctica para los profesionales de Enfermería.

En su estudio fenomenológico, Swanson observó cómo se proporcionaban los cuidados a pacientes en la UCIN; y dedujo que el proceso de los cuidados también era aplicable a madres, padres, médicos y enfermeras responsables de estos pacientes. Fue así como propuso que el cuidado integral en un ambiente complejo abarca un equilibrio de cuidados (de uno mismo y de la persona a la que se cuida), acompañamiento (a otros y roles), la asunción de responsabilidades (asignadas por uno, por otros y por la sociedad) y la evitación de malos desenlaces (28).

Con todo esto, se busca que el proceso de cuidado de Enfermería favorezca la intervención familiar a fin de minimizar el impacto emocio $\neg$ nal que se produce en la familia cuando un recién nacido ingresa a la UCIN, e impedir el deterioro del vínculo afectivo padres-hijo.

Con relación a la forma en que Swanson define las personas, los padres y sus hijos hospitalizados deben ser vistos, por parte de Enfermería, como seres únicos que están en proceso de creación y cuya integridad se completa cuando son capaces de manifestar sus pensamientos, sentimientos y conductas. Además, las experiencias vitales de madres, niños y familia están influidas por una compleja interacción de lo genético, lo espiritual y su capacidad de ejercer la autonomía, por lo cual las personas moldean y son moldeadas por el medio donde viven (29).

El personal de Enfermería debe procurar los espacios de escucha para conocer las expectativas de los padres y retroalimentar el cuidado para el bienestar, fortaleciendo, así, la comunicación padresenfermería como pilar fundamental para el cambio de paradigma que se precisa. Asimismo, a través del proceso de cuidado, el enfermero (a) debe convencer a los padres que pueden afrontar la incertidumbre causada por la separación de su hijo.

En acciones simples, como enseñar el correcto sostén del bebé por la madre, el elegir sin prisa el momento del contacto y asegurarse de que ambos se encuentren cómodos, se incorporan los 5 procesos de cuidado propuestos por la teoría de Swanson. Igualmente, hacer que las madres participen en actividades básicas de cuidado profesional como el cambio del pañal, el masaje y la hidratación de la piel, el baño y la limpieza, el cambio de tendido, entre otros, hará que el equipo de Enfermería esté realmente con los padres y posibilite el fortalecimiento del vínculo. Además, así se logrará mayor seguridad y preparación para el momento del alta y se permitirá a los padres aportar sobre las preferencias para el cuidado, a partir de sus convicciones y creencias, lo que constituye un elemento vital para el fortalecimiento del vínculo. De otra parte, todo esto hará que los padres se sientan útiles al ofrecer cuidado a sus hijos y no se perciban como un obstáculo para el equipo de Enfermería o, más en concreto, les dará mayor confianza en sí mismos, al reconocer sus capacidades y potenciales.

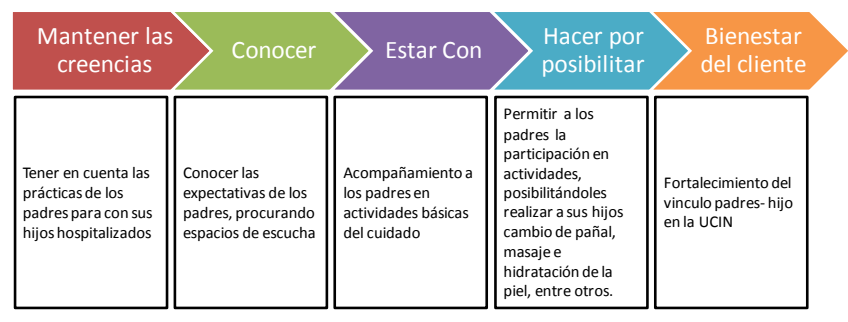

Fuente: realizado por la autora con base en SWASON, M Kristen. La Enfermería como el cuidado informado para el bienestar de los demás. J Nurs Schol. 1990; 25 (4).

Fortalecer el contacto visual y táctil entre los padres y su hijo hospitalizado, orientándolos e invitándolos a

Cienc. innov. salud. Diciembre 2013; 1 (2):120 - 125. Universidad Simón Bolívar (Col). ISSN: 2344-8636 
tocarlos sin temor, brindar el espacio y la oportunidad de intimidad, establecer estrategias de comunicación padres-hijo cuando estos no puedan permanecer en la unidad, permitiéndoles con el consentimiento de ellos y del equipo interdisciplinario de la UCIN, utilizar grabaciones con sus voces, de manera que se puedan colocar en la incubadora del neonato, podrían ser, también, estrategias de utilidad para incorporar los cinco procesos propuestos por Swanson.

\section{Conclusión}

Se requieren estudios que documenten los vacios detectados en la práctica de Enfermería $(30,31)$, así como el cuidado humanizado al interior de las UCIN. De igual forma, es fundamental que se hagan investigaciones que prueben y comprueben la eficacia y aplicabilidad de la teoría de Kristen Swanson, su estrategia de socialización y su posterior evaluación, para generar cambios en el actual sistema de atención de salud.

Por otra parte, se requiere que los profesionales de Enfermería en ejercicio mantengan el compromiso personal de crecimiento académico continuo, asistiendo a eventos de actualización y adelantado investigaciones de rigor científico y metodológico, que evalúen y muestren resultados valiosos a favor del mejoramiento continuo de la práctica de Enfermería.

Descargo de responsabilidad

El presente estudio no contó con financiación externa y no se presentan conflicto de intereses en la redacción del manuscrito..

\section{Referencias}

1. Anisfeld E, Curry M, Hales D, Kennell J, Klaus $\mathrm{M}$, O`Connor S, et al. Maternal- infant bonding: AQ joint rebuttal. Pediatrics. 1983; 72(4):569572.

2. Klauss M, Kennel J. La relación madre-hijo. Buenos Aires. Editorial Médica Panamericana. 1978.

3. Bowlby J. El vínculo afectivo. Buenos aires. 1990.

4. García SP. Cuidado neonatal con atención al desarrollo. XIX Congreso Español de Medicina Perinatal.[ Internet]. Octubre 2003. Madrid.[Citado 2013 Enero 13]; Disponible en: http://www.prematuros.cl/web-

Junio/Congresoespanol/41.cuidadodesarrollo.html

5. Franck LS, Cox S, Allen A, Winter I. Measuring neonatal intensive care unit-related parental stress. JAN. 2005; 49(6):608-615.

6. Ministerio de Salud. Resolución 13437 de 1991. Ética Hospitalaria y Decálogo de los Derechos de los Pacientes. Bogotá. 1991.

7. Martínez C. Avances y redescubrimientos en la asistencia del recién nacido Buenos Aires Argentina: Cesarini; 1996.

8. Miles MS, Funk SG. Parental Stressor Scale: neonatal intensive care unit. Nurs Res. 1993; 42 (29): 158-159.

9. Ornelas LR. Estrés de padres en la Unidad de Cuidados Intensivos Neonatales. México: Universidad Autónoma de nuevo León; 1996. Disponible en: http://cdigital.dgb.uanl.mx/te/1080072453.pdf

10. Jofre AV, Henríquez FE. Nivel de estrés de las madres con recién nacidos hospitalizados en la unidad de cuidados intensivos neonatal, Hospital Guillermo Grant Benavente de Concepción, Chile. 1999. Cienc. Enferm. 2002; 8(1):31-36.

11. Parra RD, Jirado TN. Percepciones y conductas generadas en los padres que dan cuidado especial en casa al niño atendido inicialmente en la unidad de recién nacidos del Hospital de San Ignacio, Bogotá. 1996. Invest. Educ. Enferm. 1997; 117127.

12. Hall E. Being in an alien world: Danish parents' lived experiences when a newborn or small child is critically ill. Scand J Caring Sci. 2005; 19(3):179-185.

13. Wielenga J, Smit B, Unk L. How satisfied are parents supported by nurses with the NIDCAP model of care for their preterm infant. J Nurs Care Qual. 2006; 21(1):41-48.

14. Conde AA, Belizan JM, Díaz RJ. Kangaroo mother care to reduce morbidity and mortality in low birthweight infants. Cochrane Database of Systematic Reviews 2011, Issue 3. Art. No.: CD002771.

DOI: 10.1002/14651858.CD002771.pub2.

15. Martínez J C. Atención individualizada de RN pretérmino. Rev. Hosp. Mat. Inf. Ramón Sarda. $1993 ; 12: 46-49$.

16. Helman V, Martínez A, Heath A. La presencia de la familia en la alta complejidad neonatal. Ingreso de familiares a una unidad de cuidados intensivos 
neonatales. Rev. Hosp. Mat. Inf. Ramón Sardá. 1996; 15(3): 107-112.

17. Muniagurria G. Intervenciones psicosociales en la UCIN Cuidando al recién nacido y sus padres. Simposio de seguimiento neonatal. Congreso Perinatológico Argentino. Buenos Aires, Argentina. 1998. Disponible en: http://www.psicologiaonline.com/colaboradores/gabriela/cnr.htm

18. White y col. Brazos maternos: pasado y futuro del cuidado neonatal. Clinics in Perinatology, 2004.

19. Fegran L, Helset S, Slettebo A. Nurses as moral practitioners encountering parents in neonatal intensive care units. Nurs Ethics. 2006; 13(1): 5264.

20. Ruiz CC, Muñoz RL. Cuidado de Enfermería materno perinatal en su rol asistencial, gerencial, docente e investigativo. En: Cuidado y práctica de Enfermería. Grupo de Cuidado Facultad de Enfermería. Universidad Nacional de Colombia. Bogotá, 2000.

21. Parra FF, Moncada Z, Oviedo SJ, Volcanes MM. Estrés en padres de los recién nacidos hospitalizados en la Unidad de Alto Riesgo Neonatal. Index Enferm. 2009; 18(1): 13-17.

22. Rossel CK, Carreño T, Maldonado ME. Afectividad en madres de niños prematuros hospitalizados. Un mundo desconocido. Rev. Chil. Pediatr. 2002; 73 (1): 15-21.

23. Ruiz AL, Ceriani CJ, Cravedi V, Rodríguez D. Estrés y depresión en madres de prematuros: Un programa de intervención. Arch. Argent. Pediatr. 2005; 103 (1): 36-45.

24. Ruiz A. El bebé prematuro y sus padres. Bueno Aires: Miño y Dávila Editores ED. 2004.

25. Pardo Torres MP. Familia y cuidado. En: Dimensiones del cuidado. Bogotá: Universidad Nacional de Colombia, Facultad de Enfermería; 1998, pp. 125-133.

26. Swanson KM. Nursing as Informed Caring for the Well-Being of Others. J Nurs School. 1993; 25 (4): 352-357.

27. Marriner TA. Modelos y teorías en Enfermería. Quinta edición. Editorial Elsevier. España, 2007.

28. Wojnar D. Swanson K: Teoría de los Cuidados. In: Elsevier, editor. Modelos y teorías en enfermería. Sexta ed. Madrid. España: 2007.

29. Erdmann A, Leite J, Mendes I, Trevizan M, Dantas C. Análisis de investigaciones brasileñas enfocadas en el cuidado de enfermería, años 2001-2003. Rev Cienc. Enferm. 2005; 11(2):3546.

30. Polit D, Hungler B. Investigación científica en ciencias de la salud. 6a ed. México: Gráficas Monte Albán; 2003.

31. Lincoln YS, Guba EG. Naturalistic inquiry. Beverly Hills: Sage Publications; 1985. 